\section{Islet renovation}

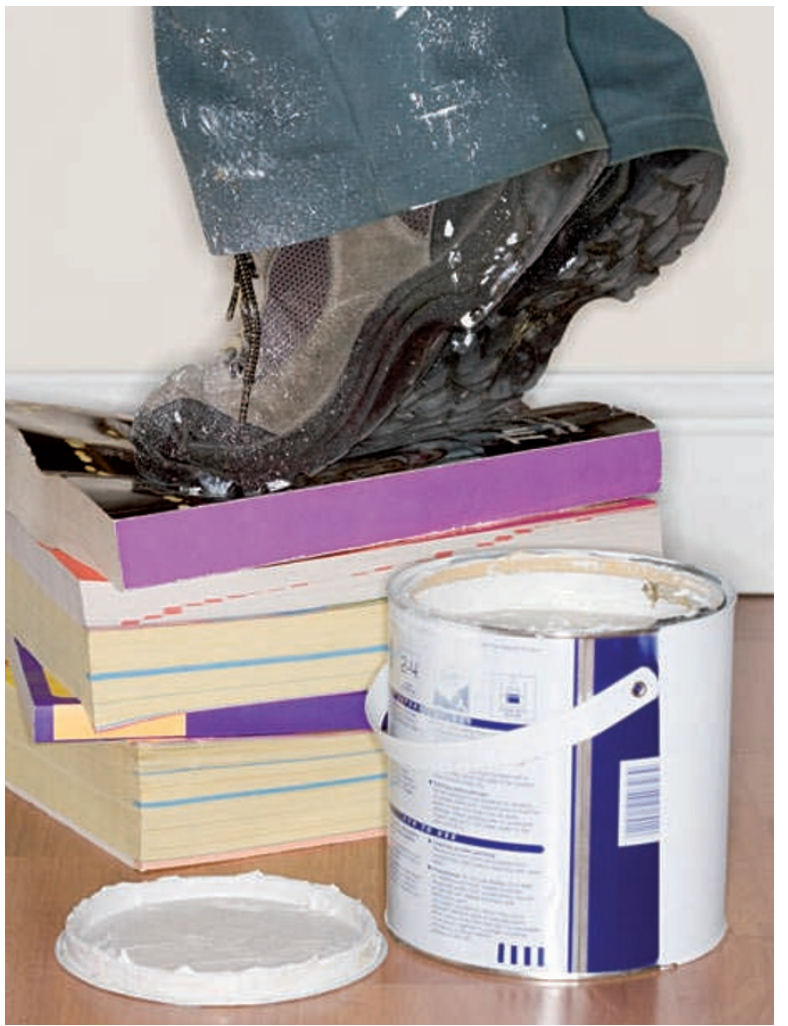

ImageSource a key role of inflammation in disease pathogenesis. Now, two papers highlight the potential therapeutic applications of human liver serine protease inhibitor $\alpha 1$ antitrypsin (AAT), an anti-inflammatory protein.

Lewis and colleagues previously reported that AAT prevented $\beta$-cell destruction and reduced inflammatory cytokines during islet transplantation in normal mice that had been rendered diabetic using streptozotocin, thereby prolonging allograft survival. Importantly, these effects were observed without the need for immunosuppression.

Now, using the same mouse model, Lewis and colleagues demonstrate that AAT also induces strain-specific immune tolerance. Twelve mice receiving AAT for 30 days or more following transplantation all accepted grafts and maintained normoglycaemia for the duration of therapy, in contrast to albumin-treated controls. Moreover, following withdrawal of AAT, continued graft-derived insulin production was observed, suggesting the induction of antigenspecific immune tolerance. This was confirmed by graft removal and retransplantation in the absence of further AAT treatment. The authors then went on to reveal that the beneficial effects of AAT were attributed to dampened expression of pro-inflammatory mediators and increased production of protective regulatory $\mathrm{T}$ cells.

In the second study, Koulmanda and colleagues propose that previous T-cell-mediated therapies have failed owing to an inability to combat nonT-cell-mediated pro-inflammatory events. To test this, they treated newly-onset non-obese diabetic (NOD) mice - a clinically relevant model of human DM1 - with AAT.
While untreated control NOD mice exhibited persistent hyperglycaemia and subsequent death, 21 out of 24 mice treated with a 15-day course of AAT attained permanent euglycaemia. Notably, $\beta$-cell mass increased from $10 \%$ in non-diabetic control mice to $45 \%$, which the authors suggest is due to repair of damaged islets, in conjunction with increased circulating fasting insulin levels. In addition, insulin resistance in insulin-sensitive tissues was ablated.

Despite exerting no direct T-cell effect, AAT also restored $\beta$-cell immune tolerance. Transplanted syngeneic islet grafts were quickly destroyed in NOD mice, but in previously euglycaemic AAT-treated NOD mice in which the diabetic state has been regenerated using streptozotocin, transplantation was successful, with all mice achieving lasting normoglycaemia. Again, further studies revealed the beneficial effects of AAT to be due to modulation of the pro-inflammatory state.

Overall, these studies highlight potential benefits of modulating inflammation in approaches to treating DM1, and importantly, safety studies for AAT have already been performed in human cases of AAT deficiency, potentially facilitating translation of this approach to clinical trials.

Sarah Crunkhorn

ORIGINAL RESEARCH PAPERS Lewis, E. C. et al. $\alpha 1$-antitrypsin monotherapy induces immune tolerance during islet allograft transplantation in mice. Proc. Natl Acad. Sci. USA 105, 16236-16241 (2008) | Koulmanda, M. et al. Curative and $\beta$ cell regenerative effects of $\alpha 1$-antitrypsin treatment in autoimmune diabetic NOD mice. Proc. Natl Acad. Sci. USA 105, 16242-16247 (2008) FURTHER READING Lewis, E. C. et al. $\alpha 1$-antitrypsin monotherapy prolongs islet allograft survival in mice. Proc. Natl Acad. Sci. USA 102, 12153-12158 (2005) 